\title{
The Use of 3D FS T1 Sequence to Diagnose Cervical Arterial Dissections in Peripartum Populations: A Case Series
}

\section{Creagh S*, Pigg N, Carr K and Shivashankar R}

Department of Radiology, Aventura Hospital and Medical Center, United States

\begin{abstract}
*Corresponding author: Susana Creagh MD, Department of Radiology, Aventura Hospital and Medical Center, 20900 Biscayne Boulevard, Aventura, Florida 33180, Tel: 7873613988; Email: scarmencr@yahoo.com
\end{abstract}

\section{Case Report \\ Volume 4 Issue 2}

Received Date: May 18, 2020

Published Date: June 29, 2020

DOI: $10.23880 /$ crij-16000172

\section{Abstract}

Cervical artery dissections (CAD) are the leading cause (20-25\%) of ischemic stroke in young and middle age adults. One particular population that is at increased risk is the peripartum woman. Imaging plays a crucial role for the initial diagnosis and follows up of CAD. Although conventional angiography is the gold standard for evaluating the arterial lumen, it doesn't offer information about the vascular wall and is unable to detect mural hematomas, thus increasing false negative rates. On the contrary, MRI imaging provides direct visualization of the mural hematoma with greater sensitivity and specificity than CT. MRI also facilitates the detection of luminal abnormalities and cerebral ischemic events resulting from CAD. Currently the 2D fat saturated (FS) T1 weighted sequence spin echo (SE) is one of the main tools in the protocol to study cervical artery dissections, yet this sequence has many limitations. Some research studies have demonstrated the advantages of the 3D T1 FS SPACE sequence(such as faster speed of acquisition, greater field of coverage and superior spatial resolution) when compared to the 2D FS T1 SE sequence. We present 3 cases of recently postpartum females who presented with neck pain, headache, and several other neurologic symptoms including stroke like symptoms and were found to have CADs on 3D FS T1 sequence MRI that were not diagnosed using conventional CT angio or ultrasound. We encourage clinicians to implement the 3D FS T1 SPACE sequence in their workup for optimal diagnosis of cervical artery dissections in the pregnant/peripartum population that present with neck pain, headache, and other neurologic symptoms, which will guide prompt treatment subsequently decreasing the rate of morbidity and mortality in this high risk population.

Keywords: Cervical Artery Dissection; MRI 3D FS T1 sequence; Peripartum

Abbreviations: CAD: Cervical Artery Dissections; MRI: Magnetic Resonance Imaging; CT: Computed Tomography; FS: Fat Saturated; SE: Spin Echo; TIA: Transient Ischemic Attack; SAH: Subarachnoid Hemorrhage; SPACE: Sequence Using Different Flip Angle Evolutions; MRA: Magnetic Resonance Angiography; RICA: right internal carotid artery; TOF: Time of flight angiography; MR: Magnetic Resonance; SPAIR: Spectral Attenuated Inversion Recovery; MPRAGE: Magnetization Prepared Rapid Gradient Echo.

\section{Introduction}

Cervical artery dissections (CAD) are the leading cause (20-25\%) of ischemic stroke in young and middle age adults. CADs often occur due to the formation of mural hematomas in the arterial walls and most commonly affect the internal carotid and vertebral arteries [1]. CAD generally happen after cervical trauma, but can also occur spontaneously in the absence of other associated risk factors such as recent infections, hypertension, and connective tissue disorders (Ehlers Danlos and Marfan syndrome) [2]. Although they can have benign clinical manifestations such as neck pain, cranial nerve palsy and Horner's syndrome, a majority of patients with CAD eventually develop TIA or stroke like symptoms [3].

One particular population that is at increased risk is the peripartum woman. It is still unclear about what factors cause increased risk within this population but multiple 
cases have been documented in the academic literature [4]. Patients can present at any time during the pregnancy or recently after and often have symptoms similar to those described above however there have been reports of stroke, subarachnoid hemorrhage (SAH), and even death [5]. Some of the mechanisms of action that have been proposed to cause CAD in the pregnant population include hormonal and mechanical factors, hypertensive disorders such as preeclampsia, and other pregnancy related etiologies [6,7].

Imaging plays a crucial role for the initial diagnosis and follows up of CAD. Although conventional angiography is the gold standard for evaluating the arterial lumen, it doesn't offer information about the vascular wall and is unable to detect mural hematomas, thus increasing false negative rates. On the contrary, MRI imaging provides direct visualization of the mural hematoma with greater sensitivity and specificity than CT [1]. MRI also facilitates the detection of luminal abnormalities and cerebral ischemic events resulting from CAD. Thus it is the imaging of choice when suspecting CAD [3].

Currently, the 2D fat saturated (FS) T1 weighted sequence spin echo (SE) is one of the main tools in the protocol to study cervical artery dissections, yet this sequence has many limitations. The implementation of a 3D FS T1 sequence using different flip angle evolutions (SPACE), has proven to have increased and possibly better value than the 2D FS T1 SE sequence on the optimal diagnosis of cervical artery dissection [8]. We present 3 cases of recently postpartum females who presented with neck pain, headache, and several other neurologic symptoms including stroke like symptoms and were found to have CADs on 3D FS T1 sequence MRI.

\section{Cases}

\section{Case 1}

The first patient is a 25 year old female without significant past medical history who presented with worsening throbbing headaches localized to the right face and back of the neck as well as into the vertex of the head 10 days after an uncomplicated, full term, spontaneous vaginal delivery. She denied nausea, vomiting or any neurological symptoms. Thorough neurological exam and vital signs were unremarkable. Imaging evaluation with bilateral carotid doppler ultrasound and CT Angiogram did not show clear evidence of dissection. Subsequent MRI and MRA however demonstrated a dissection of the cervical portion of the right carotid artery (Figure 1). The patient was managed medically with heparin and follow up CT angiogram several days later demonstrated an improved RICA dissection with interval development of a pseudoaneurysm. She was managed conservatively with Apixaban $5 \mathrm{mg}$ QD and discharged home on hospital day 5 .

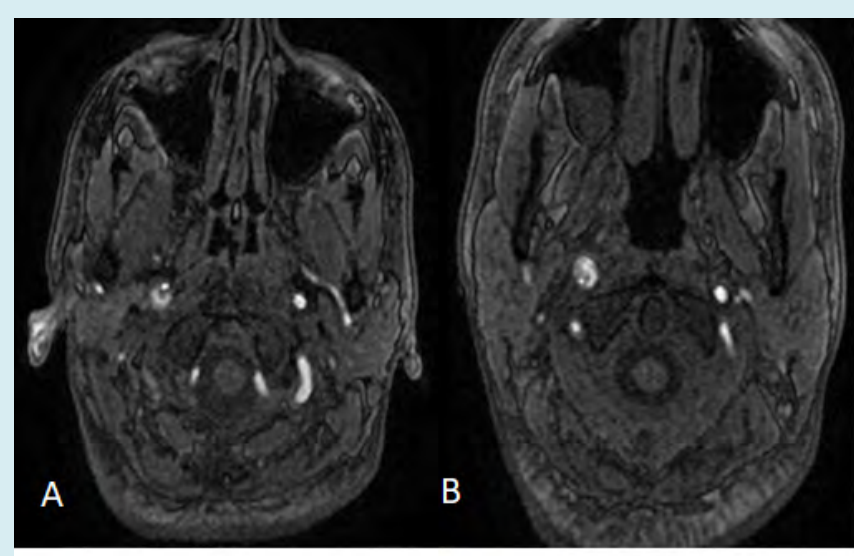

Figure 1(A\&B): Axial MRI 3D T1 FS SPACE views of the neck demonstrating T1 hyperintensity in the wall of the right carotid artery indicating a dissection of the right ICA not visualized on US or CTA.

\section{Case 2}

The second patient is a 39 year old female who developed headaches after caesarean section 6 days prior. The pregnancy was complicated by postpartum preeclampsia for which she received a standard regimen of Magnesium infusion and Ibuprofen. The patient reported a radiating cephalgia originating in the occiput and progressing down her neck. She described the pain as throbbing in character with gradual onset. She denied any history of migraines or other neurological symptoms and physical exam was non focal. Imaging evaluation with CT Angiogram demonstrated segmental narrowing of the bilateral vertebral arteries asymmetric to the left and thought to represent vasospasm (Figure 2). 


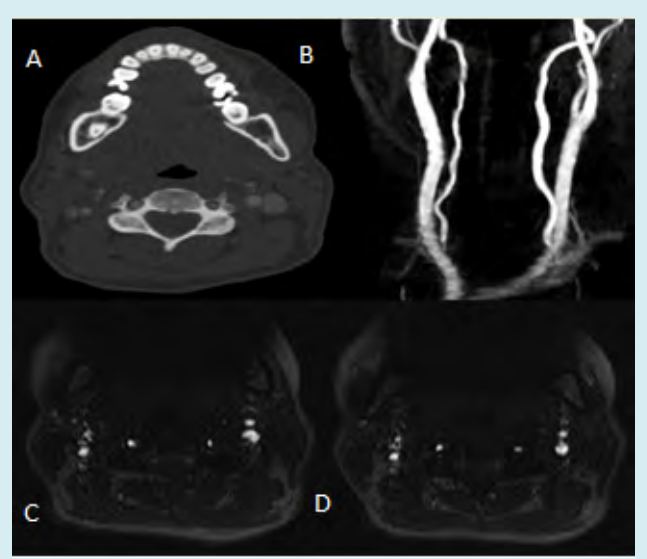

Figure 2(A-D): A-Contrast enhanced axial CT of the vertebral arteries shows narrowing of the bilateral vertebral arteries but no evidence of dissection, B-Maximum intensity projection views of the cervical arteries redemonstrate narrowing without clear dissection, C\&D-Axial MRI 3D T1 FS SPACE views of the neck demonstrating T1 hyperintensity in the wall of the bilateral vertebral arteries indicating bilateral dissection.

The patient was refractory to standard medical management for vasospasm (which included Nifedipine 60 mg daily), and continued to deteriorate. Subsequent MRI and MRA TOF imaging of the head and neck demonstrated dissections of the bilateral cervical and vertebral arteries (Figure 2). The patient was managed medical with aspirin 81 mg daily and Clopidogrel $75 \mathrm{mg}$ daily and observed clinically. Repeat imaging showed no evidence of SAH or stroke and the patient was discharged on aspirin $81 \mathrm{mg} \mathrm{QD}$ and clopidogrel $75 \mathrm{mg}$ QD and a cervical collar with instructions to follow up in several months with neurology.

\section{Case 3}

The third patient is a 36 year old female who presented with gradually worsening intractable holocephalic headaches. She presented 5 days after an uncomplicated spontaneous vaginal delivery complaining of increasing third trimester headaches during the last two weeks of the trimester. She noted intermittent, sharp left sided neck pain, left facial pain and photosensitivity. She denied additional neurological symptoms however physical exam revealed the classic triad of a left sided Horner's syndrome as well as mild weakness and sensory deficit in the left lower extremity.

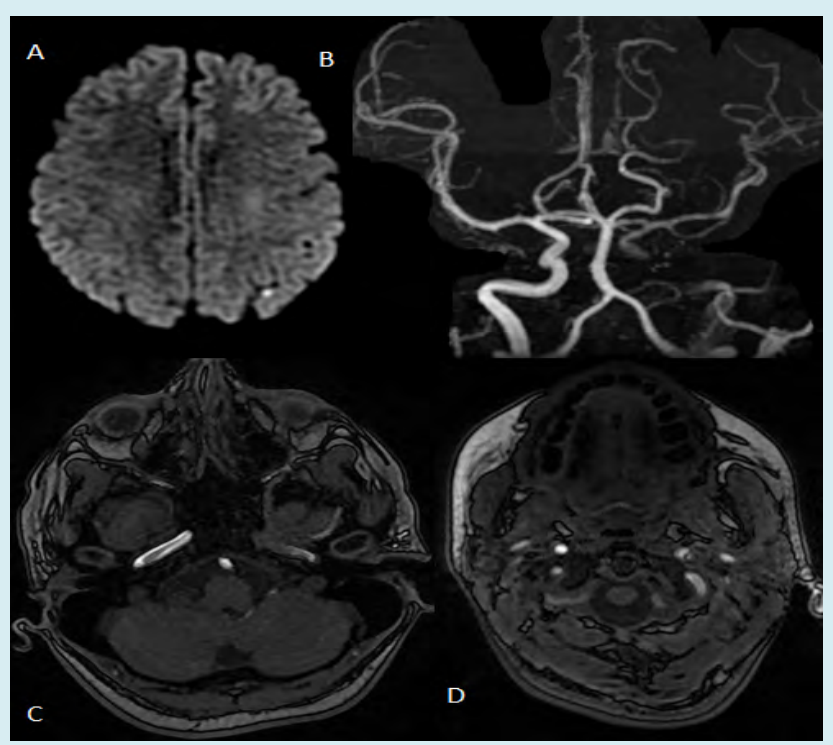

Figure 3(A-D): A-Diffusion weighted axial MRI of the brain demonstrates small are of increased activity in the posterior left parietal lobe indicating a small area of infarction, B-3D projections of the cervical arteries demonstrate nonvisualization of the left carotid artery, C\&D-Axial MRI 3D T1 FS SPACE views of the neck demonstrating T1 hyperintensity in the wall of the left carotid artery indicating a dissection of the left ICA not visualized on US or CTA. 


\section{Clinical Radiology \& Imaging Journal}

CT of the brain was non confirmatory, however MRI and MRA demonstrated a small infarct within the left posterior parietal lobe as well as an extensive, critical arterial dissection extending from the cervical to cavernous segments of the left internal carotid artery (Figure 3). No neurological intervention was attempted at this time due to pseudoaneurysm just distal to the cavernous portion of the left internal carotid artery and antiplatelet therapy was started. After continued observation and imaging the patient remained stable, and was discharged on aspirin 81mg QD, clopidogrel 75mg QD and a cervical collar with instructions to follow up with neurology for possible intervention in the future.

\section{Discussion}

Stroke is an important factor when evaluating maternal morbidity and mortality during pregnancy. The incidence of stroke in this population is currently, 4-40 strokes per 100,000 pregnancies, and it keeps uptrending when associated with comorbid conditions such as hypertension, obesity, hypercoagulable state and cardiac disease [6]. CAD is recognized as a rare etiology for ischemic stroke in pregnancy (6\%) with only about 30 cases reported in literature, mainly in the postpartum period [9].

The higher risk for CAD during pregnancy may be related to the different cardiovascular changes that take place during this period such as increase in cardiac output, increased blood flow through the carotids, and vascular compliance, which can increase shearing forces across the endothelial lining making the vessels more susceptible to intimal tear and dissection [6]. Additionally, the hypercoagulable state associated with pregnancy, can lead to the formation of thrombus at the dissection site and embolism [9].

One of the challenges, that cause delays in the clinical diagnosis of CAD during pregnancy, is the fact that the main initial symptom in nonpregnant as well as pregnant population is headache and neck pain [7]. There is a high prevalence of headaches during pregnancy associated with other conditions such as preeclampsia, eclampsia, withdrawal from caffeine, post lumbar puncture headache, and increased stress [10]. Thus the use of noninvasive imagines is crucial for prompt diagnosis under a strong clinical suspicion for CAD, since immediate therapy with anticoagulation should be initiated to prevent/minimize damage, particularly with ischemic lesions [10].

Currently, the 2D fat saturated (FS) T1 weighted sequence spin echo (SE) is one of the main tools in the protocol to study cervical artery dissections. Fat suppression is crucial to be able to differentiate subacute intramural hemorrhage from surrounding fat tissue since both produce hyperintense signal. Although this technique is effective, it has many limitations: it is time consuming to cover all neck and head area and is suboptimal on arteries with tortuous course [6]. The implementation of a 3D FS T1 sequence using different flip angle evolutions (SPACE) has proven to have added and possibly better value to the 2D FS T1 SE sequence on the optimal diagnosis of cervical artery dissection [6]. The 3D T1 FS SPACE main parameters consists of 56 slices with 256 × 256 matrix (with a 512 × 512 interpolation), echo time $22 \mathrm{~ms}$, repetition time 750, variable flip angle, a field of view of $23 \times 23 \mathrm{~cm}$ and acquisition time of $3 \mathrm{~min} 25 \mathrm{sec}$ [11].

Some research studies have already demonstrated the advantages of the 3D T1 FS SPACE sequence when compared to the 2D FS T1 SE sequence. This sequence is able to cover all arterial portions (cervical and intracranial) in a single acquisition and with specific parameters being SPAIR fat saturation mode, averages 1.4 shorter acquisition time (3mins) when compared to the 2S FS T1 SE (11min) [12]. This provides the advantage to detect all lesions in just one acquisition in a shorter MR protocol time [12]. Additionally this sequence allows multiplanar reformatting that clearly delineate the hyper intensity of the intramural hematoma which facilitates the assessment of the extension of the dissection, particularly when looking at tortuous arterial segments such as the carotid petrous entry or the atlas loop of the vertebral artery that have been previously missed by 2D FS T1 SE [13]. It also makes it easier to analyze vascular loops, particularly in the V3 segment of the vertebral artery. In addition it facilitates reproducible comparisons with gadolinium enhanced MRA [8].

The T1 FS SE sequence has noted limitations in terms of quality in the presence of magnetic susceptibility artifacts. Previous case reports have demonstrated the ability of 3D T1 FS SPACE sequence to overcome magnetic susceptibility artifacts from dental implants, surgical metallic hardwares as well as movement artifacts $[14,15]$. This enhances optimal analysis of the extension of the dissections. Moreover, it is also known that the quality of fat suppression is suboptimal at lower cervical levels, a limitation that is also overcome by 3D T1 FS SPACE sequence [14]. Other advantages of the 3D T1 FS SPACE sequence reported in literature are its higher sensitivity for detecting tumors in the cervical region, MS brain lesions and leptomeningeal abnormalities when compared to other sequences such as the T1 MPRAGE while allowing reformatting in any plane $[11,16]$.

\section{Conclusion}

Our cases highlight the advantages of adding the 3D T1 FS SPACE sequence to the protocol for the diagnosis of cervical artery dissection in women during pregnancy and postpartum period under the appropriate clinical setting. 


\section{Clinical Radiology \& Imaging Journal}

We encourage physicians to consider ordering the SPACE MRI study for any woman during terminal pregnancy or puerperium period that presents with persistent headaches, neck pain and associated neurological symptoms such as ipsilateral Horner syndrome or TIA, particularly with underlying risk factors such as preeclampsia, hypertension and obesity, since they would be at high risk of having cervical artery dissection. With its many advantages (such as faster speed of acquisition, greater field of coverage and superior spatial resolution), 3D SPACE MRI will speed early and accurate diagnosis of this entity, facilitate prompt treatment, prevent associated ischemic events and subsequently decrease the rate of morbidity and mortality in this high risk population.

Disclosure: The authors report no conflict of interest concerning the materials of methods used in this manuscript. This research was supported (in whole or in part) by HCA and/or an HCA affiliated entity. The views expressed in this publication represent those of the author(s) and do not necessarily represent the official views of HCA or any of its affiliated entities. There were no external sources of financial support for this manuscript.

\section{References}

1. Hassen WB, Machet A, Edjlali-Goujon M, Legrand L, Ladoux A, et al. (2014) Imaging of Cervical Artery dissection. Diagn Interv Imaging 95(12): 1151-1161.

2. Rubinstein SM, Peerdeman SM, van Tulder MW, Riphagen I, Haldeman S (2005) A systematic review of the risk factors for cervical artery dissection. Stroke 36(7): 1575-1580.

3. Debette S, Leys D (2009) Cervical artery dissections: predisposing factors, diagnosis, and outcome. Lancet Neurol 8(7): 668-678.

4. Mohammed I, Aaland M, Khan N, Crossley I (2014) A young pregnant woman with spontaneous carotid artery dissection--unknown mechanisms. BMJ Case Rep.

5. Tuluc M, Brown D, Goldman B (2006) Lethal Vertebral Artery Dissection in Pregnancy: A Case Report and Review of the Literature. Arch Pathol Lab Med 130(4): 533-535.

6. Reinhard M, Munz M, Kannen AL, Griesser-Leute HJ, Dittrich R, et al. (2014) Risk of recurrent cervical artery dissection during pregnancy, childbirth and puerperium.
Eur J Neurol 22(4): 736-739.

7. Shanmugalingam R, Pour NR, Chuah SC, Vo MT, Beran R, et al. (2016) Vertebral artery dissection in hypertensive disorders of pregnancy: a case series and literature review. BMC Pregnancy Childbirth 16(1): 164.

8. Bisseret D, Khalil A, Favrole P, Carette MF, Marro B (2014) Spontaneous cervical artery dissection: Role of a SE- T1 weighted fat sat volume acquisition. Diagn Interv Imaging: 95(4): 443-446.

9. Ulrich N, Johonson A, Jodry D, Dola C, Martin-Schild S, et al. (2015) Resolution of Internal Carotid Dissection with Middle Cerebral Artery Occlusion in Pregnancy. Case Rep Neurol Med 2015: 398261.

10. Maderia LM, Hoffman MK, Shlossman PA (2007) Internal Carotid Artery Dissection as a Cause of Headache in the Second Trimester. American J Obstetrics \& Gynecology 196(1): 7-8.

11. Jeevanandham B (2015) To compare post contrast 3D T2 FLAIR, T1-SPACE and MP-RAGE sequences to select the ideal sequence for leptomeningeal abnormalities at 3T MRI. ECR 2015.

12. Cuvinciuc V, Viallon M, Momjian-Mayor I, Sztajzel R, Pereira VM, et al. (2013) 3D fat saturated T1 SPACE sequence for the diagnosis of cervical artery dissection. Neuroradiology 55(5): 595-602.

13. Yang $Q$, Guo X, Fan Z, Wan M, Li D (2015) Cervial artery dissection: value of 3D high resolution vessel wall magnetic resonance imaging for diagnosis and followup. J Cardiovasc Magn Reson 17(1): 412.

14. Bencheikh Y, Kulczycka P, Macri F, Millon D, Greffier J, et al. (2016) Interest of 3D Fat Saturated T1 Weighted Magnetic Resonance Imaging for the Assessment of Cervical Artery Dissection. J Belg Soc Radiol 100(1): 27.

15. Cha JG, Jin W, Lee MH, Kim DH, Park JS, et al. (2011) Reducing metallic artifacts in postoperative spinal imaging: usefulness of IDEAL contrast-enhanced T1 and T2 weighted MR imaging-phantom and clinical studies. Radiolgy 259(3): 885-893.

16. Paret M, Doussin S, Barral FG, Boutet C (2015) Reliable imaging of Neurovasculature with Dot and T1 SPACE. Clinical Neurology 63(3): 2015. 